\title{
The effect of differential galactic winds on the chemical evolution of galaxies
}

\author{
S. Recchi ${ }^{1,2}$, E. Spitoni ${ }^{3}$, F. Matteucci ${ }^{1,3}$, and G. A. Lanfranchi ${ }^{4}$ \\ 1 INAF - Osservatorio Astronomico di Trieste, via G.B. Tiepolo 11, 34131, Trieste, Italy \\ e-mail: [recchi;matteucc]@oats.inaf.it \\ 2 Institute of Astronomy, Vienna University, Türkenschanzstrasse 17, 1180 Vienna, Austria \\ 3 Department of Astronomy, Trieste University, via G.B. Tiepolo 11, 34131 Trieste, Italy \\ e-mail: spitoni@oats.inaf.it \\ 4 Núcleo de Astrofísica Teórica, Universidade Cruzeiro do Sul, R. Galvão Bueno 868, Liberdade, 01506-000, São Paulo, SP, Brazil \\ e-mail: gustavo.lanfranchi@unicsul.br
}

Received 31 March 2008 / Accepted 28 July 2008

\section{ABSTRACT}

\begin{abstract}
Aims. The aim of this paper is to study the basic equations of the chemical evolution of galaxies with gas flows. In particular, we focus on models in which the outflow is differential, namely in which the heavy elements (or some of the heavy elements) can leave the parent galaxy more easily than other chemical species such as $\mathrm{H}$ and $\mathrm{He}$.

Methods. We study the chemical evolution of galaxies in the framework of simple models, namely we make simplifying assumptions about the lifetimes of stars and the mixing of freshly produced metals. This allows us to solve analytically the equations for the evolution of gas masses and metallicities. In particular, we find new analytical solutions for various cases in which the effects of winds and infall are taken into account

Results. Differential galactic winds, namely winds carrying out preferentially metals, have the effect of reducing the global metallicity of a galaxy, with the amount of reduction increasing with the ejection efficiency of the metals. Abundance ratios are predicted to remain constant throughout the whole evolution of the galaxy, even in the presence of differential winds. One way to change them is by assuming differential winds with different ejection efficiencies for different elements. However, simple models apply only to elements produced on short timescales, namely all by type II SNe, and therefore large differences in the ejection efficiencies of different metals are unlikely.

Conclusions. Variations in abundance ratios such as $[\mathrm{O} / \mathrm{Fe}]$ in galaxies, without including the $\mathrm{Fe}$ production by type Ia supernovae, can in principle be obtained by assuming an unlikely different efficiency in the loss of $\mathrm{O}$ relative to Fe from type II supernovae. Therefore, we conclude that it is not realistic to ignore type Ia supernovae and that the delayed production of some chemical elements relative to others (time-delay model) remains the most plausible explanation for the evolution of $\alpha$-elements relative to Fe.
\end{abstract}

Key words. galaxies: abundances - galaxies: evolution - galaxies: ISM

\section{Introduction}

When a stellar system is formed out of gas, the dying stars enrich the remaining gas so that new stars are metal-enriched compared to their ancestors. Simple models of chemical evolution have been used since decades to interpret the evolution of the chemical composition of stars and gas. Pioneering works have determined the evolution of the metallicity in the Galaxy (Schmidt 1963; Searle \& Sargent 1972; Tinsley 1974; Pagel \& Patchett 1975) by making very simplifying hypotheses about the initial mass function (IMF), the lifetime of stars and the mixing of chemical elements with the surrounding interstellar medium (ISM).

These models remain useful guides for understanding the main chemical properties of galaxies, but it was soon realized that they cannot give a complete picture of galaxy evolution. In particular, simple closed box models cannot reproduce the distribution of metallicity of long-living stars in the galaxy ( $G$ - $d$ warf problem, Lynden-Bell 1975; Rana 1991, and references therein). The most obvious solution to the G-dwarf problem is to relax the hypothesis that the solar neighborhood evolved as a closed box and to allow for infall of gas (Tinsley 1980). Increasingly complex models of chemical evolution including gas flows (infall, outflow, radial flows), but always assuming the instantaneous recycling approximation (IRA) ${ }^{1}$, have been proposed, covering a large variety of galactic morphologies (Larson 1976; Clayton 1988; Edmunds 1990; Köppen 1994; Martinelli \& Matteucci 2000). These models consider the global metallicity $Z$ and neglect metallicity-enriched outflows, so they cannot be used to predict variations of abundance ratios. Pagel \& Tautvaišienė (1998) have calculated abundance ratios using analytical models, but they had to assume that chemical elements such as iron are ejected with a fixed time delay $\Delta$ after the time of star formation. This is a rough approximation since it is known that the progenitors of SNeIa span a very wide lifetime (see e.g. Matteucci $\&$ Recchi 2001). Only detailed numerical models relaxing IRA and including the chemical enrichment from type Ia SNe (e.g. Matteucci \& Greggio 1986) allow one to follow in detail the evolution of single elements. Matteucci \& Greggio (1986) showed in detail the effect of the time-delay model, already suggested by Tinsley (1980) and Greggio \& Renzini (1983), in particular the effect of a delayed Fe production by type Ia SNe on abundance ratios involving $\alpha$-elements $(\mathrm{O}, \mathrm{Mg}, \mathrm{Si})$.

\footnotetext{
1 IRA assumes that stars with masses larger than $1 M_{\odot}$ die instantaneously whereas those below $1 M_{\odot}$ live forever (Matteucci 2001).
} 
Outflows and galactic winds are produced when the thermal energy of a galaxy (produced by energetic events such as $\mathrm{SN}$ explosions and stellar winds) is enough to unbind a large fraction of the ISM (Mathews \& Baker 1971). The existence of differential winds, namely galactic winds in which the metals (or some of them) are ejected out of the parent galaxy more efficiently than the other elements, has been first hypothesized and applied to the chemical evolution of galaxies by Pilyugin (1993) and Marconi et al. (1994). In particular, Marconi et al. (1994) suggested that the chemical properties of gas-rich dwarf irregular galaxies, most remarkably the spread in $\mathrm{H} / \mathrm{He}$ vs. $\mathrm{O} / \mathrm{H}$ and $\mathrm{N} / \mathrm{O}$ vs. $\mathrm{O} / \mathrm{H}$, can be more easily explained by assuming differential winds, in which products of SNeII (mostly $\alpha$-elements and one third of iron) can escape the potential well of a galaxy more easily than other elements formed in low and intermediate mass stars. Hereafter we will call selective winds the differential winds in which different metals have different ejection efficiencies. The existence of differential and selective winds has been theoretically confirmed by several detailed chemodynamical simulations (MacLow \& Ferrara 1999; D’Ercole \& Brighenti 1999; Recchi et al. 2001; Fujita et al. 2004) and it has been observationally confirmed by determining the metallicity of outflows in starburst galaxies (Martin et al. 2002; Ott et al. 2005). Recently, Salvadori et al. (2008) have suggested differential winds coupled with relaxation of IRA in massive stars to explain the steep decline of the $[\mathrm{O} / \mathrm{Fe}]$ ratio observed in the stars of Sculptor, without invoking any Fe production from type Ia SNe.

In this paper we apply the hypothesis of differential and selective winds in the framework of simple models of chemical evolution with IRA. The chemical evolution of galaxies is a complex phenomenon that can be properly treated only by means of detailed numerical codes, possibly taking into account also the hydrodynamical evolution of the system, as we have explained above. However, in spite of the oversimplifications introduced by simple chemical evolution models, the results they produce are very linear and simple to interpret. They can therefore illustrate in a direct way the effect of differential winds on the chemical evolution of galaxies, as well as the effect of relaxing IRA. Moreover, for clarity purposes, we show also the results of detailed numerical models (Lanfranchi \& Matteucci 2003, 2004) as a comparison.

The plan of the paper is as follows: in Sect. 2 we summarize the assumptions of the simple models of chemical evolution and the results of models including gas flows (infall and outflow). In Sect. 3 we introduce the assumptions necessary to solve the equations of chemical evolution of galaxies with differential winds and produce some illustrative result. In Sect. 4 we study the abundance ratios of some chemical elements predicted by models with differential and selective winds. Finally, we discuss some implications of the results and draw the main conclusions in Sect. 5.

\section{Summary of simple model containing gas flows}

\subsection{Standard solutions}

As it is well known, the so-called Simple Model of chemical evolution is based on the following assumptions:

1. the system is one-zone and closed, namely there are no inflows or outflows;

2. the initial gas is primordial (no metals);

3. the IMF is constant in time;

4. the gas is well mixed at any time (instantaneous mixing);
5. stars more massive than $1 M_{\odot}$ die instantaneously; stars smaller than $1 M_{\odot}$ live forever (instantaneous recycling approximation or IRA).

These simplifying assumptions allow us to calculate analytically the chemical evolution of the galaxies. Once we have defined the fundamental quantities:

$R=\int_{1}^{\infty}\left(m-M_{R}\right) \phi(m) \mathrm{d} m$,

(where $\phi(m)$ is the IMF and $M_{R}$ is the mass of the remnant) and

$y_{Z}=\frac{1}{1-R} \int_{1}^{\infty} m p_{Z, m} \phi(m) \mathrm{d} m$

(where $p_{Z, m}$ is the fraction of newly produced and ejected metals by a star of mass $m$ ), the well known solution of the closed box model can be easily found:

$Z=y_{Z} \ln \left(\mu^{-1}\right)$

where $\mu$ is the gas fraction $M_{\mathrm{g}} / M_{\mathrm{tot}}$. This result is obtained by assuming that the galaxy initially contains only gas and has the remarkable property that it does not depend on the particular star formation history the galaxy experiences. To obtain Eq. (3) one has to assume that $y_{Z}$ does not depend on metallicity. Nucleosynthesis can indeed depend on metallicity, especially for secondary elements. However, for $\mathrm{O}$ and $\mathrm{Fe}$ the metallicity effect is not so important (Matteucci 2001; Garnett 2002), therefore we can neglect it for the purposes of our work. In the general case in which $y_{Z}=f(Z)$ the evolution of the metallicity is governed by the following equation:

$\int_{0}^{Z} \frac{\mathrm{d} w}{f(w)}=\ln \left(\mu^{-1}\right)$.

Relaxing the first of the assumptions of the simple model, we get the models including gas flows, also known as leaky box models. Analytical solutions of simple models of chemical evolution including infall or outflow are known since at least 30 years (Pagel \& Patchett 1975; Hartwick 1976; Twarog 1980; Edmunds 1990). Here we follow the approach and the terminology of Tinsley (1980) and Matteucci (2001), namely we assume for simplicity linear flows (we assume gas flows proportional to the star formation rate (SFR)). Therefore, the outflow rate $W(t)$ is given by:

$W(t)=\lambda(1-R) \psi(t)$,

where $\psi(t)$ is the SFR, and the infall rate $A(t)$ is given by:

$A(t)=\Lambda(1-R) \psi(t)$.

Here $\lambda$ and $\Lambda$ are two proportionality constants. The first assumption is justified by the fact that, the larger the SFR is, the more intense are the energetic events associated with it (in particular supernova explosions and stellar winds), therefore the larger is the chance of having a large-scale outflow (see e.g. Silk 2003). A proportionality between $A(t)$ and $\psi(t)$ can be instead justified by the fact that the infall of gas provides a continuous reservoir for the star formation. A careful analysis of this assumption is given in Sect. 3.4.

The system of equations we need to solve is therefore:

$\left\{\begin{array}{l}\frac{\mathrm{d} M_{\text {tot }}}{\mathrm{d} t}=(\Lambda-\lambda)(1-R) \psi(t) \\ \frac{\mathrm{d} M_{\mathrm{g}}}{\mathrm{d} t}=(\Lambda-\lambda-1)(1-R) \psi(t) \\ \frac{\mathrm{d} M_{Z}}{\mathrm{~d} t}=(1-R) \psi(t)\left[\Lambda Z_{A}+y_{Z}-(\lambda+1) Z\right]\end{array}\right.$ 


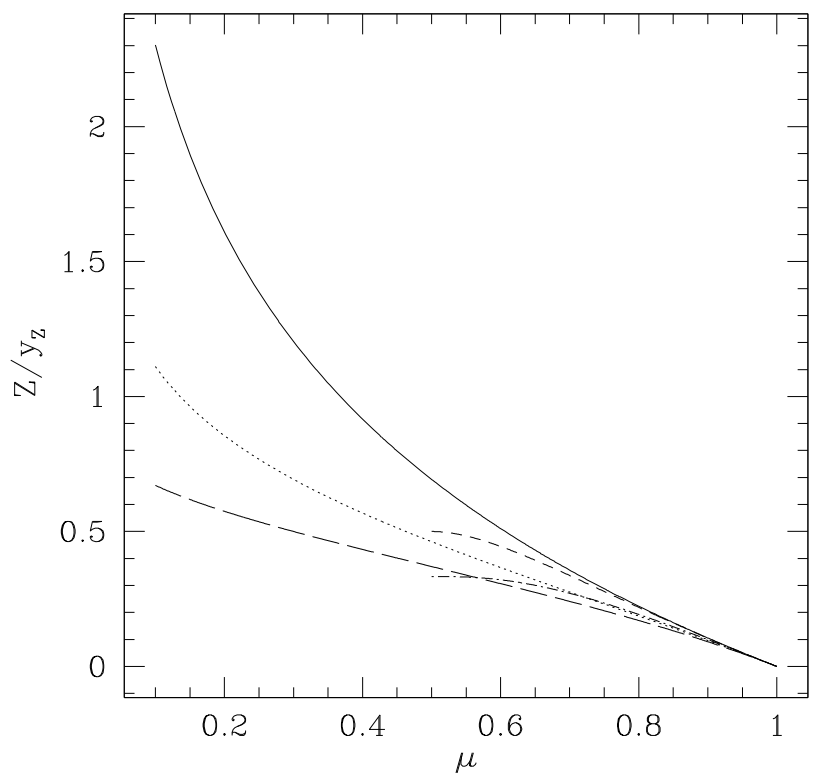

Fig. 1. Evolution of the metallicity as a function of $\mu$ for the closed box model (solid line) and models with gas flows: $\lambda=2$ and $\Lambda=0$ (dotted line); $\lambda=0$ and $\Lambda=2$ (short-dashed line); $\lambda=3$ and $\Lambda=1$ (longdashed line); $\lambda=1$ and $\Lambda=3$ (dot-dashed line). The metallicity of the infalling gas $Z_{A}$ is assumed to be 0 .

where $M_{Z}$ is the mass of metals $\left(M_{Z}=Z \cdot M_{\mathrm{g}}\right)$ and $Z_{A}$ is the metallicity of the infalling gas. For the moment, $Z_{A}$ is assumed to be a constant, although it could change with time due to the pollution of the intracluster medium (ICM) by means of the metals ejected through the galactic winds. This aspect will be considered in detail in Sects. 3.2 and 3.3. With some algebra, this set of equations can be worked out, yielding:

$Z=\frac{\Lambda Z_{A}+y_{Z}}{\Lambda}\left\{1-\left[(\Lambda-\lambda)-(\Lambda-\lambda-1) \mu^{-1}\right]^{\frac{\Lambda}{\Lambda-\lambda-1}}\right\}$.

To find this solution we have made use of the initial conditions $Z(0)=0, M_{\mathrm{g}}(0)=M_{\mathrm{tot}}(0)=M_{\mathrm{g}, 0}$. It is worth pointing out that the explicit dependence of $Z$ on time is hidden in the time dependence of $\mu$ (see also Sect. 3.4).

We show in Fig. 1 the evolution of $Z / y_{Z}$ versus $\mu$ in the case $Z_{A}=0$ for different values of $\lambda$ and $\Lambda$. It is worth reminding that $\mu=1$ at the beginning of the evolution of the galaxy and its value decreases with time. Therefore, the time axis is inverted compared to the $\mu$-axis in this figure and in similar ones. We can see that all the models with gas flows produce metallicities smaller than the ones predicted by the closed box model (solid line). This is in agreement with the first and the third of Edmund's theorems (Edmunds 1990), since we have assumed that the infall gas is pristine $\left(Z_{A}=0\right)$. Figure 2 shows the effective yields $y_{\text {eff }}=Z / \ln \left(\mu^{-1}\right)$ (normalized to $y_{Z}$ ) of the same models presented in Fig. 1. $y_{\text {eff }}$ is often used in literature to interpret data and to analyze to what extent the chemical evolution of a galaxy deviates from the behavior predicted by the closed box model (Garnett 2002; Tremonti et al. 2004). We can notice from Eq. (8) that the solution diverges for models in which $\lambda>\Lambda$ when $\mu$ approaches 0 , therefore, we show $Z / y_{Z}$ and $y_{\text {eff }} / y_{Z}$ versus $\mu$ only until $\mu \sim 0$.1. Indeed, Prantzos \& Aubert (1995) pointed out that IRA cannot be applied in situations in which low $\mu$ are obtained, because the amount of gas ejected by low mass stars at late times can substantially modify the final abundances, also for elements produced on short timescales by massive stars. We notice here also that, in models in which the infall rate is larger than the

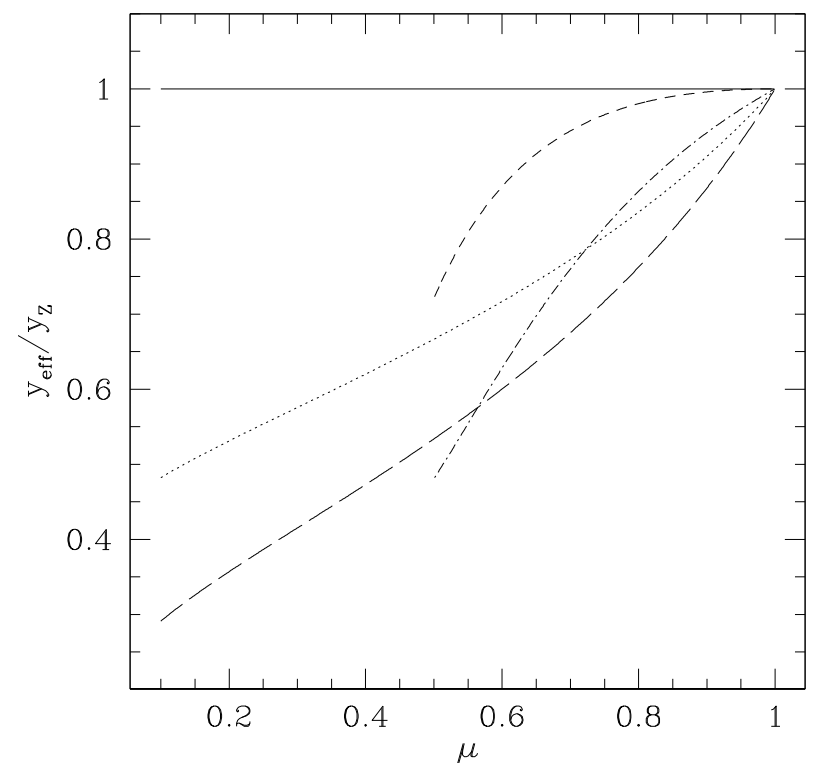

Fig. 2. Normalized effective yield as a function of $\mu$. Notation as in Fig. 1.

outflow rate, not all the values of $\mu$ are allowed. Solving the first two equations of the system (7), we get:

$\mu=\frac{\Lambda-\lambda-1}{\Lambda-\lambda}+\frac{1}{\Lambda-\lambda} \frac{M_{\mathrm{g}, 0}}{M_{\mathrm{g}}}$

In the models in which $\Lambda>\lambda+1, M_{\mathrm{g}}$ is always increasing (Eq. (7)), therefore $\mu$ ranges between 1 and a minimum value:

$\mu_{\min }=\frac{\Lambda-\lambda-1}{\Lambda-\lambda}$.

For models in which $\lambda+1>\Lambda>\lambda$ there is no $\mu_{\text {min }}$ but there is a upper limit reachable by the gas mass which is given by $M_{\mathrm{g}, \text { lim }}=M_{\mathrm{g}, 0} /(\lambda+1-\Lambda)$. For this reason we plotted in Figs. 1 and 2 the model with $\lambda=0$ and $\Lambda=2$ (short-dashed line) and the model with $\lambda=1$ and $\Lambda=3$ (dot-dashed line) only for $\mu \geq \mu_{\min }=0.5$.

\section{Simple models with differential winds}

\subsection{The basic solution}

We present in this section the chemical evolution of galaxies in which a differential wind is assumed, namely in which the metals are more easily channelled out of the parent galaxy than the pristine gas. The easiest way to consider a differential wind in the framework of simple models of chemical evolution is to assume that the metallicity of the gas carried out in the galactic wind is proportional to the metallicity of the ISM with a proportionality constant larger than one. If we define $Z^{\circ}$ as the metallicity of the outflowing gas, this condition implies that $Z^{\circ}=\alpha Z$ with the ejection efficiency $\alpha>1$. In the metallicity budget (third equation in (7)) we have to assume that the negative term due to the galactic wind is given by $W(t) Z^{\circ}=\alpha \lambda(1-R) \psi(t)$. A similar approach has been used by Salvadori et al. (2008) but in the framework of a semi-analytical model for the evolution of galaxies. With our simple approach we are able to determine analytical expressions for the evolution of $Z$, which allow us to understand more clearly the effect of galactic winds on the chemical evolution of galaxies. 


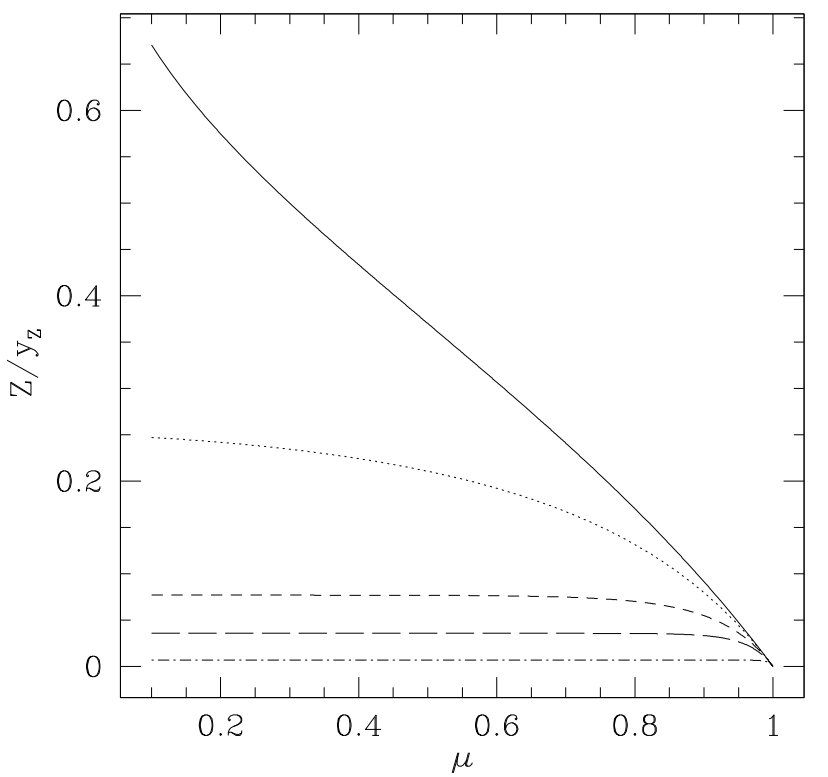

Fig. 3. Evolution of the metallicity as a function of $\mu$ for simple models with differential winds, $\lambda=3, \Lambda=1$ and $Z_{A}=0$. Plotted are models with the differential wind parameter $\alpha=2$ (dotted line), 5 (short-dashed line), 10 (long-dashed line) and 50 (dot-dashed line). For reference, also the model with $\alpha=1$ (normal wind) is plotted (solid line).

The set of the equations we have to solve in this case is very similar to (7), with the only difference given by the metallicity budget equation, which we modify as follows:

$\frac{\mathrm{d} M_{Z}}{\mathrm{~d} t}=(1-R) \psi(t)\left[\Lambda Z_{A}+y_{Z}-(\lambda \alpha+1) Z\right]$.

The solution of this new set of equations is given by:

$Z=\frac{\Lambda Z_{A}+y_{Z}}{\Lambda+(\alpha-1) \lambda}\left\{1-\left[(\Lambda-\lambda)-(\Lambda-\lambda-1) \mu^{-1}\right]^{\frac{\Lambda+(\alpha-1) \lambda}{\Lambda-\lambda-1}}\right\}$.

It is straightforward to see that we can obtain Eq. (8) in the case $\alpha=1$ (i.e. in the case in which the galactic wind is not differential). We remind here that this solution is valid only for primary elements. The evolution of secondary elements (namely elements synthesized from the metals present in the stars at birth) in the framework of simple models with gas flows has been extensively studied by Köppen \& Edmunds (1999) and we refer the reader to this paper to find out more about it.

Assuming again for simplicity an infall of pristine gas (i.e. $Z_{A}=0$ ) we can see how the chemical evolution of the galaxy varies with the ejection efficiency $\alpha$. In Figs. 3 and 4 we show $\mathrm{Z} / y_{Z}$ vs. $\mu$ for different values of $\alpha$ and gas flow parameters $(\lambda, \Lambda)$ taken as $(3,1)$ and $(1,3)$, respectively. The first couple of values can be attributed to objects in which galactic winds have a prominent role. This is usually the case in dwarf galaxies, since the potential well of these objects is very shallow and large-scale outflows must be common (Dekel \& Silk 1986; but see also Skillman 1997). The second set of parameters $(\lambda=1$, $\Lambda=3$ ) can more properly refer to spiral galaxies, although large outflow episodes in massive spirals are not very likely. In general, the effect of differential winds can be more clearly distinguished in models in which the outflow is prominent compared to the infall. For this reason, we will pay from now on more attention on the models for which $\lambda>\Lambda$, taking $\lambda=3, \Lambda=1$ as

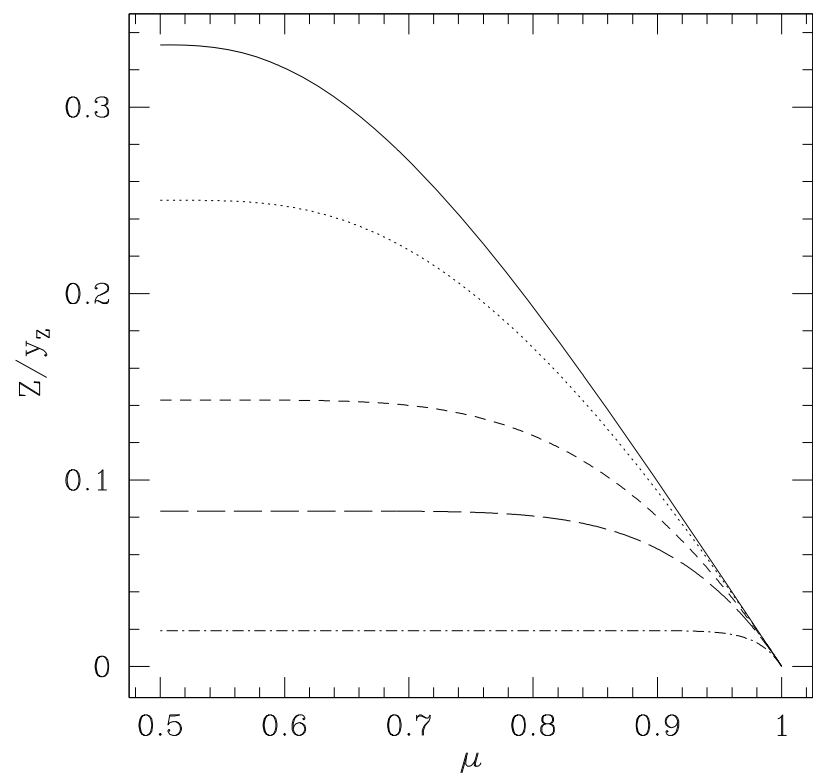

Fig. 4. As in Fig. 3 but for $\lambda=1$ and $\Lambda=3$.

reference values. Our aim in this paper is not to describe a prototypical dwarf galaxy, therefore the combination of parameters $(\lambda, \Lambda)=(3,1)$ is purely indicative and serves as a benchmark of our models with differential winds.

As expected, the differential wind determines a significant reduction of the metallicity of the galaxy. It can be shown analytically that, for each value of $\alpha>1$, Eq. (12) produces metallicities systematically smaller that the ones obtained from Eq. (8). Moreover, it is evident from these plots the asymptotic trend of $Z / y_{Z}$ as $\mu$ approaches 0 or $\mu_{\min }$. From Eq. (12) it is easy to see that, in both cases, $Z / y_{Z}$ asymptotically tends to the value $[(\alpha-1) \lambda+\Lambda]^{-1}$ provided that $Z_{A}=0$, therefore $Z / y_{Z}$ decreases almost linearly with $\alpha$ when $\mu$ is small and $\alpha \gg 1$.

\subsection{Instantaneous ICM mixing models}

In our calculations we have so far assumed that the metallicity of the infalling gas $Z_{A}$ is constant in time. This assumption is not necessarily true, in particular when differential winds are taken into account. In fact, the galactic winds can pollute the surrounding medium and the infalling gas can have a metallicity increasing with time. The increase of the iron abundance between redshift $z \sim 1$ and $z \sim 0$ in the ICM has been recently suggested (Balestra et al. 2007) and can be accounted for in numerical models in which the chemical evolution of the ICM is affected by galactic winds and stripping (Calura et al. 2007). A simple hypothesis we can make about the variation of the ICM metallicity with time is that the metals, expelled out of the galaxy through galactic winds, instantaneously and uniformly mix with the surrounding ICM. This approach has been used by detailed chemical evolution models of the ICM (e.g. Matteucci \& Vettolani 1988; Moretti et al. 2003). Of course the instantaneous mixing of the outflowing gas with the whole ICM is rather unlikely. Nevertheless, this assumption is useful for illustrative purposes. This hypothesis leads to a new set of equations in which, besides $Z_{A}$, we have to consider also the variation, although small, 


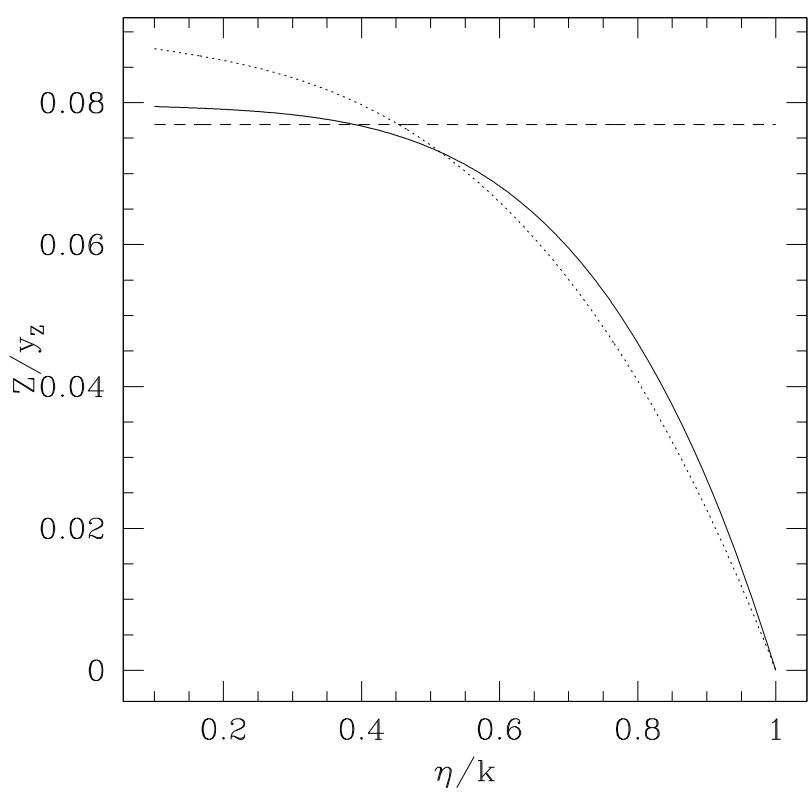

Fig. 5. Chemical evolution of models in which the metallicity of the ICM varies with time (instantaneous ICM mixing models) and with initial mass ratio $k$ (see text) equal to 0.1 (solid line) and 0.5 (dotted line). Also shown in this plot is the asymptotic trend of the simple differential wind model for $\mu$ approaching 0 (dashed line). For all models, $\lambda=3$, $\Lambda=1$ and $\alpha=5$.

of the ICM mass $M_{\mathrm{ICM}}$ due to galactic winds and infall. These 4 equations are:

$\left\{\begin{array}{l}\frac{\mathrm{d} M_{\mathrm{g}}}{\mathrm{d} t}=(\Lambda-\lambda-1)(1-R) \psi(t) \\ \frac{\mathrm{d} M_{Z}}{\mathrm{~d} t}=(1-R) \psi(t)\left[\Lambda Z_{A}+y_{Z}-(\lambda \alpha+1) Z\right] \\ \frac{\mathrm{d} M_{Z_{A}}}{\mathrm{~d} t}=(1-R) \psi(t)\left[\lambda \alpha Z-\Lambda Z_{A}\right] \\ \frac{\mathrm{d} M_{\mathrm{I} C}}{\mathrm{~d} t}=(1-R) \psi(t)(\lambda-\Lambda),\end{array}\right.$

where $M_{Z_{A}}$ is the mass of metals in the $\operatorname{ICM}\left(M_{Z_{A}}=Z_{A} \cdot M_{\mathrm{ICM}}\right)$. In order to solve this set of equations we have to define the ratio between gas mass and ICM mass $\eta=M_{\mathrm{g}} / M_{\mathrm{ICM}}$ and a new parameter $k=M_{\mathrm{g}, 0} / M_{\mathrm{ICM}}(0)$, where $M_{\mathrm{ICM}}(0)$ is the initial ICM mass. Once we have defined the values $X_{a}$ and $X_{b}$ such that:

$X_{a}^{-1}=\frac{\lambda-\Lambda}{(k+1)(\lambda-\Lambda)+1} \eta-\frac{\Lambda-\lambda-1}{(k+1)(\lambda-\Lambda)+1}$,

and

$X_{b}^{-1}=\frac{k(\lambda-\Lambda)}{(k+1)(\lambda-\Lambda)+1}-\frac{k(\Lambda-\lambda-1)}{(k+1)(\lambda-\Lambda)+1} \eta^{-1}$,

the solution of the system of Eqs. (13) is given by:

$Z=\frac{y_{Z}}{\Lambda \alpha X_{a}^{\frac{\lambda}{\Lambda-\lambda}}-(\Lambda-\lambda)(\alpha-1)}\left[1-X_{b}^{-\frac{\Lambda \alpha X_{a}^{\frac{\lambda}{\Lambda-\lambda}}-(\Lambda-\lambda)(\alpha-1)}{\Lambda-\lambda-1}}\right]$.

It is straightforward to see that Eqs. (16) and (12) produce the same results if the infall is negligible (i.e. if $\Lambda \sim 0$ ). Considering again a model with $\Lambda=1$ and $\lambda=3$ we can show the trend of $Z / y_{Z}$ in the case $\alpha=5$ for different values of $k$. We notice first that, unlike $\mu, \eta$ does not monotonically decrease, but its variation depends on the values of $\lambda$ and $\Lambda$. In the present example, since $\lambda>\Lambda, M_{\mathrm{g}}$ decreases with time and $M_{\mathrm{ICM}}$ increases with time, therefore $\eta$ ranges between $k$ at the beginning and 0 during the late stages of the evolution of the galaxy. We show in Fig. 5 the evolution of $Z / y_{Z}$ as a function of $\eta / k$ for $k=0.1$ (solid line) and $k=0.5$ (dotted line). The model with $k=0.5$ predicts larger abundances because the metals carried out by the galactic wind are diluted with less ICM, therefore $Z_{A}$ is larger. These results cannot be compared to the results of Eq. (12) in a straightforward manner since they are expressed in different units. However, we show in this plot the asymptotic trend of the simple differential wind model (short-dashed line in Fig. 3) for $\mu$ approaching 0 (dashed line). This comparison allows us to notice that, as expected, Eq. (16) predicts larger metallicities compared to models with $Z_{A}=0$. However, as long as $k<1$, the differences are small (of the order of $\sim 0.1-0.2$ dex).

We can also calculate the ICM metallicity, which turns out to be:

$Z_{A}=\alpha Z\left(1-X_{a}^{\frac{\lambda}{\Lambda-\lambda}}\right)$

In the case we are considering more closely, $\lambda=3$ and $\Lambda=1$, therefore the ICM mass increases with time and $X_{a}$ ranges between 1 and $(1+k)$. The larger value $Z_{A} / Z$ can attain is therefore:

$\frac{Z_{A}}{Z}=\alpha\left[1-(1+k)^{\frac{\lambda}{\Lambda-\lambda}}\right]$

which is smaller than 1 provided that:

$k<k_{\lim }=\left[\frac{\alpha}{\alpha-1}\right]^{\frac{\lambda-\Lambda}{\lambda}}-1$.

Once the ratio $Z_{A} / Z$ between ICM and ISM metallicity is observationally known, Eq. (18) can be used to constrain $\alpha$.

\subsection{Galactic fountain models}

A special case of variable infall metallicity is represented by the situation in which $Z_{A}=Z^{0}=\alpha Z$, namely the metallicity of the infalling gas is set to be always equal to the one of the galactic wind. This condition implies therefore that the very same gas that has been driven out of the galaxy by energetic events can subsequently rain back to the galaxy, due to the pull of its gravitational potential. This kind of duty cycle is well known in astrophysics and it has been named galactic fountain (Shapiro \& Field 1976; Bregman 1980). In order to solve the chemical evolution in this special case, we have necessarily to assume that $\lambda \geq \Lambda$ since the reservoir for the infall gas is given by the gas expelled out of the galaxy through galactic winds. The solution of the chemical evolution in the presence of galactic fountains is given by:

$Z=\frac{y_{Z}}{(\lambda-\Lambda)(\alpha-1)}\left\{1-\left[(\Lambda-\lambda)-(\Lambda-\lambda-1) \mu^{-1}\right]^{\frac{(\lambda-\Lambda)(\alpha-1)}{\Lambda-\lambda-1}}\right\}$

A comparison of this solution with the solution of Eq. (12) with infall of pristine gas $\left(Z_{A}=0\right)$ is shown in Fig. 6 . For both models plotted in this figure we have assumed $\lambda=3, \Lambda=1$ and $\alpha=5$. As expected, the galactic fountain model predicts larger vales of $Z$. These values exceed also the metallicities attained by the models with instantaneous mixing of the ICM (Sect. 3.3) shown in Fig. 5. This result is expected because the galactic fountain model maximizes the enrichment in metallicity of the infalling gas, whereas in the models shown in Sect. 3.3 the metals escaping from the galaxy are diluted in a large amount of ICM. From Eqs. (12) and (20) we can also notice that the metallicity ratio tends asymptotically to the value $[(\alpha-1) \lambda+\Lambda] /[(\lambda-\Lambda)(\alpha-1)]$ which is always larger than one (for the special choice of parameters it is equal to $13 / 8$ ) but it tends to 1 for $\alpha \gg 1$. In 


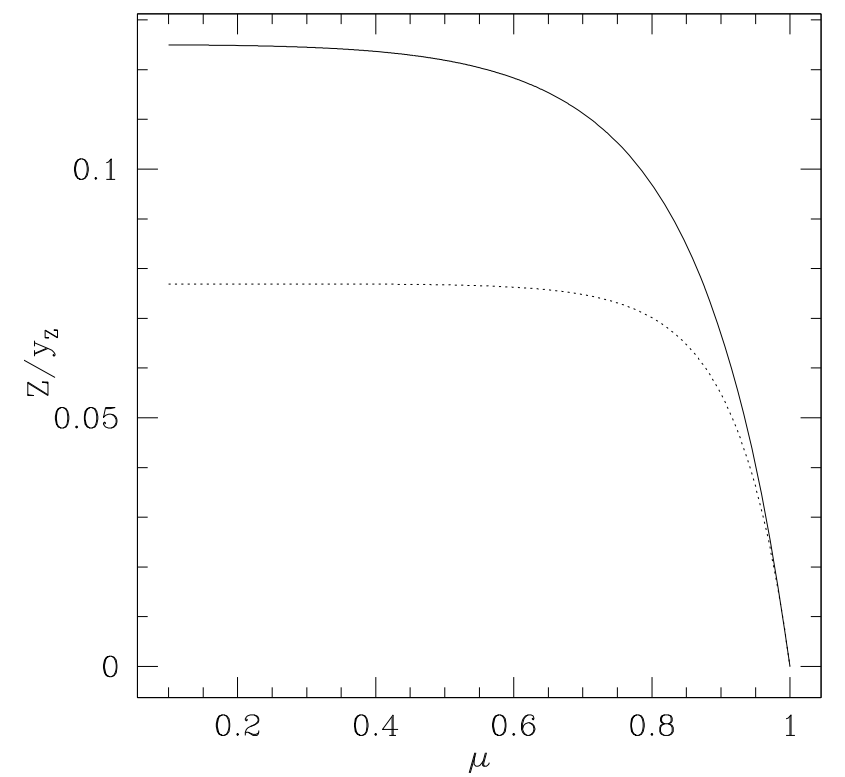

Fig. 6. Comparison of the chemical evolution of a galactic fountain model (solid line) with a model with infall of pristine gas $\left(Z_{A}=0\right.$, dotted line). For both models, $\lambda=3, \Lambda=1$ and $\alpha=5$.

our example, only an infall of pre-enriched gas, whose metallicity is larger than $5 / 8 y_{Z}$ can therefore produce a metallicity in the galaxy larger than the one attained by the galactic fountain model. From Eq. (20) we can also see that, in the special case in which $\lambda=\Lambda, Z / y_{Z}$ tends to the solution of the closed box model (Eq. (3)). This is due to the fact that, in the framework of the simple models of chemical evolution, having outflow and infall with the same rate and metallicity or not having gas flows at all is formally the same. Indeed, the fountains take a finite and non-negligible time to orbit around and fall back to the galaxy (Spitoni et al. 2008). This implies a delay in the mixing of metals in the ISM, which conflicts with the fourth assumption of the simple models of chemical evolution (Sect. 2), therefore only detailed numerical models can ascertain the effect of this delay on the chemical evolution of galaxies (Spitoni et al. 2008, in preparation).

\subsection{Limits of the approximations of linear flows}

In the previous sections we have assumed that both outflow and infall rates are proportional to the SFR and we have seen that in this way we obtain solutions in which $\psi(t)$ cancels out. In this section we analyze how reliable are these assumptions and what kind of results we obtain if we assume general infall and outflow laws (see also Edmunds 1990; Köppen \& Edmunds 1999). First of all we notice that a correlation between infall rate and SFR arises naturally because the larger is the amount of infalling gas, the higher is the reservoir of gas inside the galaxy available to form stars. An example is given by detailed models of the chemical evolution of the Milky Way (Chiappini et al. 1997; Cescutti et al. 2007; Colavitti et al. 2008) in which two main phases of gas infall turn out to produce two distinct episodes of star formation (although the similar behavior between the two rates does not imply that their ratio is constant). We will show in this section that, under reasonable assumptions, a constant ratio between SFR and infall rate can be attained during the late evolution of the considered galaxies. On the other hand, a proportionality between the outflow rate and the SFR is quite realistic and it has been shown both observationally (e.g. Heckman 2002) and theoretically (e.g. Silk 2003).

Therefore, let us assume for simplicity that Eq. (5) still holds and that the infalling gas is pristine (e.g. $\left.Z_{A}=0\right)$. The extension of our calculations to the case in which $W(t)$ is a generic function is straightforward. If we assume a generic infall law $A(t)$, the system of equations we need to solve is:

$$
\left\{\begin{array}{l}
\frac{\mathrm{d} M_{\text {tot }}}{\mathrm{d} t}=A(t)-\lambda(1-R) \psi(t) \\
\frac{\mathrm{d} M_{\mathrm{g}}}{\mathrm{d} t}=A(t)-(\lambda+1)(1-R) \psi(t) \\
\frac{\mathrm{d} M_{Z}}{\mathrm{~d} t}=(1-R) \psi(t)\left[y_{Z}-(\alpha \lambda+1) Z(t)\right],
\end{array}\right.
$$

subject to the usual initial conditions $Z(0)=0, M_{\mathrm{g}}(0)=$ $M_{\mathrm{tot}}(0)=M_{\mathrm{g}, 0}$. In this case, however, it is not possible to solve this system of equations canceling out $\psi(t)$. Therefore, at variance with what we have done so far, we must assume some dependence of $\psi(t)$ on $M_{\mathrm{g}}$ (Schmidt law). We assume a linear Schmidt law (e.g. $\psi(t)=S M_{\mathrm{g}}(t)$ ), because it is the only formulation for which the results can be expressed analytically. For Schmidt laws with different exponents detailed numerical models are required. The equation for the evolution of the gas mass can be simply solved, yielding:

$$
M_{\mathrm{g}}(t)=\mathrm{e}^{-(\lambda+1)(1-R) S t}\left[\int_{0}^{t} A(u) \mathrm{e}^{(\lambda+1)(1-R) S u} \mathrm{~d} u+M_{\mathrm{g}, 0}\right] .
$$

Substituting this expression into the third equation of the system (21) also the evolution of the metallicity as a function of time can be calculated. The result is the following:

$Z(t)=(1-R) S y_{Z}[I(t)]^{-1} \int_{0}^{t} I(u) \mathrm{d} u$

where

$I(t)=\mathrm{e}^{\lambda(1-R) S(\alpha-1) t+\int_{o}^{t} \frac{A(u) \mathrm{e}^{(\lambda+1)(1-R) S u}}{M_{\mathrm{g}, 0^{+}} \int_{0}^{u} A(v) \mathrm{e}(\lambda+1)(1-R) S v \mathrm{~d} v} \mathrm{~d} u}$.

We consider now as an example an exponential infall law, study what kind of metallicity evolution it produces and compare the results with those presented in Sect. 3.1, bearing in mind that, in the present case, we have to define the value of some constants $\left(M_{\mathrm{g}, 0}, S\right.$ as well as the constants defining the infall rate), whereas in the previous case it was enough to define the proportionality constant $\Lambda$. We have:

$A(t)=K \mathrm{e}^{-t / t_{1}}=K \mathrm{e}^{-\tau}$,

where $t_{\mathrm{i}}$ is a characteristic infall timescale and $\tau=t / t_{\mathrm{i}}$. We can calculate $K$ assuming that the initial gas mass $M_{\mathrm{g}, 0}$ is proportional to the total amount of infalling gas through a proportionality constant $\gamma$ (namely we assume that $M_{\mathrm{g}, 0}=\gamma \int_{0}^{\infty} A(t) \mathrm{d} t$ ). In this way, we obtain $K=\frac{M_{\mathrm{g}, 0}}{\gamma t_{\mathrm{i}}}$. Equation (22) can be therefore solved:

$M_{\mathrm{g}}(\tau)=\frac{M_{\mathrm{g}, 0}}{K_{1}}\left[\mathrm{e}^{-\tau}+K_{1} \mathrm{e}^{-(\lambda+1)(1-R) S t_{\mathrm{i}} \tau}\right]$,

where $K_{1}$ is a constant (see the Appendix for its exact value).

We can now evaluate the ratio $\Lambda(t)=\frac{A(t)}{(1-R) \psi(t)}$ in order to test if and under which conditions we can still assume a proportionality between the SFR and the infall rate $A(t)$. In Fig. 7 we show the resulting $\Lambda(t)$ for different values of $\gamma\left(0.2,1\right.$ and 5) and $t_{\mathrm{i}}$ (1, 1.5 and $5 \mathrm{Gyr})$. In order to draw these curves we have assumed $\lambda=3$ (in agreement with what assumed in Sect. 3.1) and 


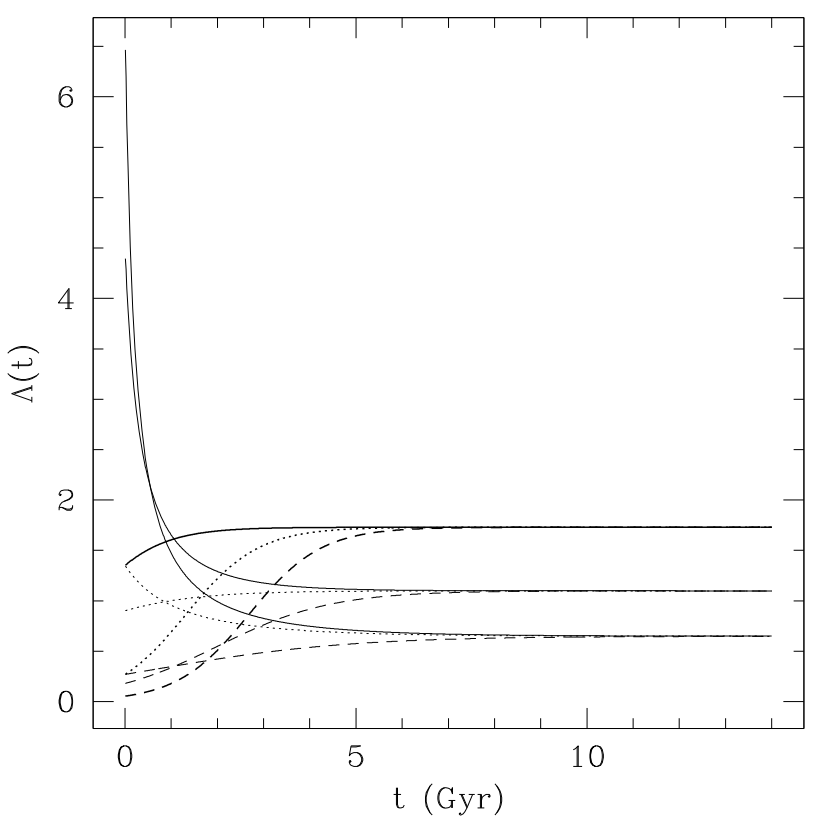

Fig. 7. Ratio between the infall rate and the SFR as a function of time for models with an exponential infall and different $t_{\mathrm{i}}$ and $\gamma$ (see text). Heavy lines: $t_{\mathrm{i}}=5 \mathrm{Gyr}$; normal lines: $t_{\mathrm{i}}=1.5 \mathrm{Gyr}$; light lines: $t_{\mathrm{i}}=$ 1 Gyr. Solid lines: $\gamma=0.2$; dotted lines: $\gamma=1$; dashed lines: $\gamma=5$.

$S=1 \mathrm{Gyr}^{-1}$. This is an upper limit for the star formation efficiency in dwarf galaxies (see e.g. Lanfranchi \& Matteucci 2003). $R$ is assumed to be 0.26 , in agreements with the nucleosynthetic calculations of Woosley \& Weaver (1995) assuming a Salpeter IMF. A small value of $\gamma$ implies that the galaxy initially does not have a large amount of gas and the star formation is mostly triggered by the infalling gas, therefore the infall rate initially prevails over the SFR. A large $t_{\mathrm{i}}$ implies a large infall rate at late times, therefore $\Lambda(t)$ tends asymptotically to a large value. In general the behavior of $\Lambda(t)$ depends on a complex interplay between $\gamma, t_{\mathrm{i}}, S$ and $\lambda$ (see also Köppen \& Edmunds 1999) but we can see that under reasonable assumptions the models show a first phase (lasting a few Gyr) in which $\Lambda(t)$ varies with time, but then it stabilizes to a constant value. As a general result, only if we assume infall timescales much shorter than the star formation timescale (i.e. the inverse of $S$ ) $\Lambda(t)$ shows significant variations over the whole evolution of the galaxy, although these variations are milder if a large value of $\lambda$ is assumed. For instance, if we take $S=0.1 \mathrm{Gyr}^{-1}$, a constant $\Lambda(t)$ is attained by assuming either large values of $\lambda$ or large values of $t_{\mathrm{i}}$ or values of $\gamma$ such that $K_{1} \sim 0$. If we assume a quadratic Schmidt law, the infall timescales required to obtain a constant $\Lambda(t)$ tend to be larger than in the case of a linear Schmidt law. We can conclude that a linear flow can be a reasonable approximation of the late evolution of a galaxy provided that the infall timescale is of the same order of magnitude of the star formation timescale.

Now we can answer the following question: does an early phase of variable $\Lambda(t)$ significantly affect the evolution of the metallicity? To do that we have to solve Eq. (23). The resulting evolution of $Z / y_{Z}$ is given by:

$\frac{Z(\tau)}{y_{Z}}=\frac{K_{2}+K_{3} \mathrm{e}^{E_{1} \tau}-\left(K_{2}+K_{3}\right) \mathrm{e}^{-E_{2} \tau}}{\mathrm{e}^{E_{1} \tau}+K_{1}}$,

(see the Appendix for the expressions of the involved constants).

We show in Fig. 8 the evolution of $Z(t) / y_{Z}$ as a function of time for different values of $\gamma$. In these models we assume

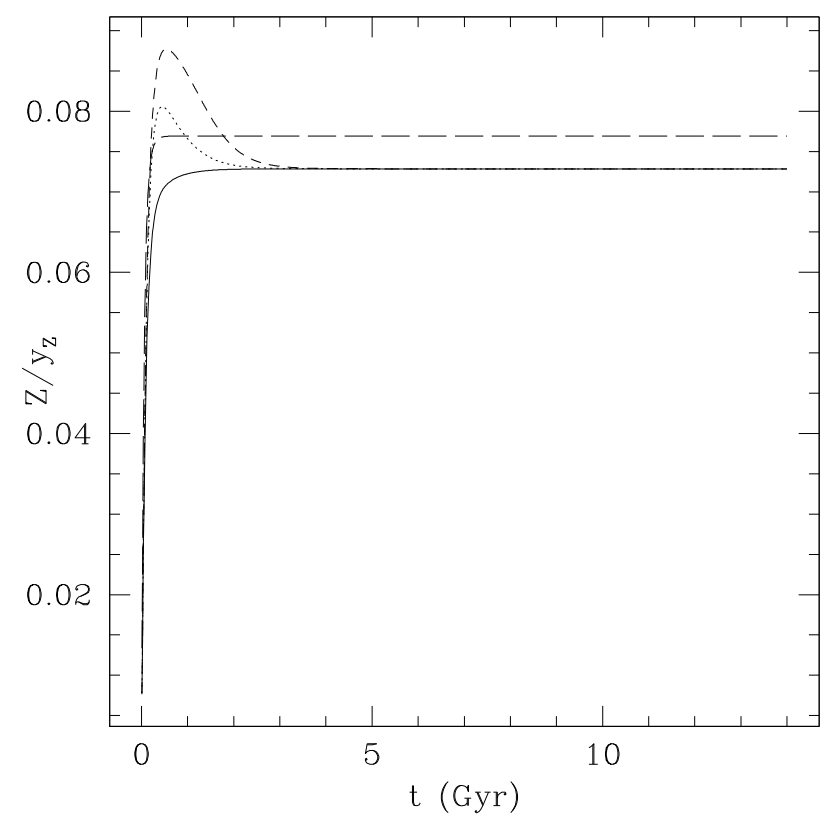

Fig. 8. Evolution of the metallicity as a function of time for models with an exponential infall and different $\gamma$. Solid line: $\gamma=0.2$; dotted line: $\gamma=1$; short-dashed line: $\gamma=5$. For all the models it is assumed $t_{\mathrm{i}}=1.5 \mathrm{Gyr}$. Also plotted (long-dashed line) the evolution of a model in which a linear infall (with $\Lambda=1$ ) is assumed. For all models $\alpha=5$.

$t_{\mathrm{i}}=1.5 \mathrm{Gyr}$ because this value leads to a $\Lambda$ close to 1 (see Fig. 7). To compare these models with the results shown in Sect. 3.1 we combine the first two equations of the system (7) to get $\mu(t)$ in a model with linear flows and a linear Schmidt law. By means of Eq. (12) we then obtain the evolution of $Z$ as a function of time, which turns out to be:

$\frac{Z(t)}{y_{Z}}=\frac{1-\left[(\Lambda-\lambda+1)-(\Lambda-\lambda) \mathrm{e}^{(\lambda+1-\Lambda)(1-R) S t}\right]^{\frac{\Lambda+(\alpha-1) \lambda}{\Lambda-\lambda-1}}}{\Lambda+(\alpha-1) \lambda}$

We plotted also this curve in Fig. 8 for $\Lambda=1$ (long-dashed line). The difference with the models with exponential infall laws is very small and mostly due to the fact that the asymptotic value of $\Lambda(t)$ for the exponential infall models is not exactly 1 . Therefore we can conclude that the results we have shown in Sect. 3.1 do not change substantially if we assume an exponential infall instead of an infall rate proportional to the SFR. This demonstrates also that the major source of error in the solutions of the simple models is the IRA assumption rather than the assumption of linear flows.

\section{The abundance ratios}

From Eq. (12) it is straightforward to determine the abundance ratio between two chemical elements $i$ and $j$ assuming that the yields do not depend on metallicity (see also Sect. 2), namely:

$\frac{Z_{i}}{Z_{j}}=\frac{y_{i}+\Lambda Z_{A, i}}{y_{j}+\Lambda Z_{A, j}}$

This abundance ratio is therefore determined uniquely by constants which depend on the IMF and on the properties of the infalling gas. In the models with variable infall metallicities, $Z_{i} / Z_{j}$ is simply given by the yields ratio $y_{i} / y_{j}$ (see Eqs. (16) and (20)). With our simplifying assumptions, the abundance ratio must remain constant throughout the whole evolution of the 
galaxy. Also the abundance ratios of the ICM (see Eq. (17)) must remain constant with $\eta$ (and therefore with time). The constancy of abundance ratios in the framework of simple models of chemical evolution was also pointed out by Matteucci \& Chiappini (2005), in which the authors showed that variations of the abundance ratios can be accounted for only by relaxing the IRA and by properly taking into account stellar lifetimes. Here we outline that this result holds also if we consider differential winds.

By neglecting the contribution of SNeIa, it is expected that the $[\mathrm{O} / \mathrm{Fe}]$ ratio does not change with time (and therefore with $[\mathrm{Fe} / \mathrm{H}]$ ) since, in this case, both elements are produced by SNeII, on similar timescales. Indeed, detailed models of the evolution of the Galaxy (Chiappini et al. 1997) show a mild decrease of the predicted (and observed) $[\mathrm{O} / \mathrm{Fe}]$ as a function of $[\mathrm{Fe} / \mathrm{H}]$ even without considering SNeIa, due to the variation of the yields with stellar mass, but this can account for only $\sim 0.2$ dex of the $[\mathrm{O} / \mathrm{Fe}]$ variation. A much more significant reduction of the $[\mathrm{O} / \mathrm{Fe}]$ can be attained by taking into consideration SNeIa, which provide most of the Fe of the ISM and explode with a delay compared to the SNeII. This model (also called time-delay model) remains the most plausible explanation of the variation of $[\mathrm{O} / \mathrm{Fe}]$ vs. $[\mathrm{Fe} / \mathrm{H}]$ in various galaxies (Matteucci \& Greggio 1986; Lanfranchi \& Matteucci 2003). In particular, Lanfranchi \& Matteucci (2003) interpreted the steep decline of the $[\alpha / \mathrm{Fe}]$ ratios at low $[\mathrm{Fe} / \mathrm{H}]$ (at $[\mathrm{Fe} / \mathrm{H}] \sim-1.6 \mathrm{dex})$, observed in dwarf spheroidals of the Local Group (e.g. Shetrone et al. 2001; Venn et al. 2004) as due to the slow SFR acting in these systems, coupled with very efficient galactic winds. In this situation, in fact, the Fe production from type Ia $\mathrm{SNe}$ occurs when the $[\mathrm{Fe} / \mathrm{H}]$ is still low. At this point, the galactic winds extract both $\alpha$-elements and Fe from the galaxy but while $\mathrm{O}$ is less and less produced because of the decrease in the star formation due to the wind, Fe continues to be produced by type Ia SNe. Unfortunately, SNeIa cannot be treated by means of simple model (SNeIa arise from $\mathrm{CO}$ white dwarfs in binary stars, with non-negligible lifetimes, therefore the IRA cannot be applied). Because of that, it is not possible to solve analytically chemical evolution equations which include SNeIa and this is the reason why most people make use of numerical simulations. However, IRA provides a good approximation of the evolution of $\alpha$ elements, promptly released by SNeII. Recently, Savadori et al. (2008) proposed an alternative scenario in which the drop in the $[\mathrm{O} / \mathrm{Fe}]$ is due to strong differential winds, combined with the relaxation of the IRA, without invoking SNeIa. In their model, the star formation is dominated by an early, very short ( $100 \mathrm{Myr})$ burst. However, a non-negligible fraction of SNeIa seems to appear also within 0.1 Gyr in galaxies (see Mannucci et al. 2005, 2006), therefore neglecting SNeIa in their model could be incorrect. This might be the reason why these authors do not reproduce accurately the high metallicity tail of the metallicity distribution function of Sculptor (namely the stars with $[\mathrm{Fe} / \mathrm{H}] \sim-1$; see their Figs. 4 and 6). On the other hand, neglecting type Ia $\mathrm{SNe}$ appears consistent with the stellar lifetimes they have assumed. These lifetimes predict $150 \mathrm{Myr}$ for a $8 M_{\odot}$ star, instead of 30-40 Myr as commonly adopted. These long lifetimes, together with the short duration of the main episode of SF and the relaxation of IRA help in producing the decrease of $[\mathrm{O} / \mathrm{Fe}]$ since in this case the evolution of abundance ratios resembles that of an instantaneous burst in which the $[\mathrm{O} / \mathrm{Fe}]$ at a given time $t$ follows the ratio of $\mathrm{O}$ and $\mathrm{Fe}$ yields of the star dying at that time.

One way to attain a variable $[\mathrm{O} / \mathrm{Fe}]$ ratio in the framework of simple models of chemical evolution is by assuming selective winds, namely that the two elements have different ejection efficiencies. It has been suggested since a long time that $\alpha$-elements

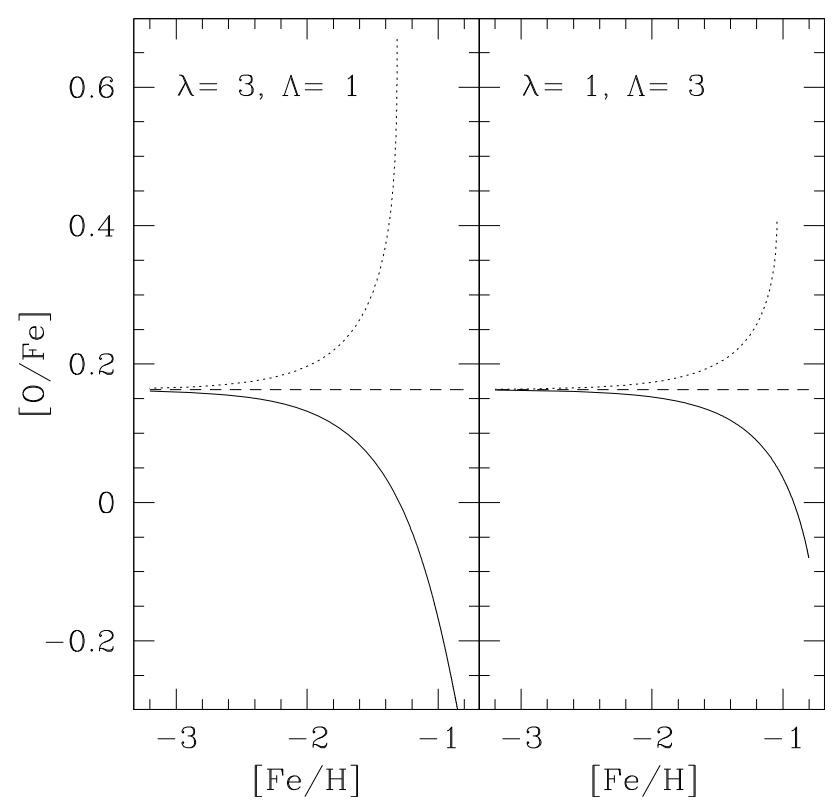

Fig. 9. $[\mathrm{O} / \mathrm{Fe}]$ vs. $[\mathrm{Fe} / \mathrm{H}]$ for models characterized by different ejection efficiencies for $\mathrm{O}$ and $\mathrm{Fe} \alpha_{\mathrm{O}}$ and $\alpha_{\mathrm{Fe}} .\left(\alpha_{\mathrm{O}}, \alpha_{\mathrm{Fe}}\right)$ are $(5,2)$ (solid line), (2, $5)$ (dotted line) and $(2,2)$ (dashed line). $(\lambda, \Lambda)$ are $(3,1)$ (left panel) and $(1,3)$ (right panel).

can be channelled out of a galaxy more easily than other elements (Marconi et al. 1994; Martin et al. 2002) but Recchi et al. (2001) showed that, during the very first burst of star formation experienced by a dwarf galaxy, iron can be expelled more easily than oxygen. The hypothesis of different ejection efficiencies $\alpha$ for $\mathrm{O}$ and $\mathrm{Fe}$ is therefore a reasonable assumption that can be tested by our models.

We assume two different values for these ejection efficiencies $\alpha_{\mathrm{O}}$ and $\alpha_{\mathrm{Fe}}$ and, by means of Eq. (12), we calculate how [O/Fe] varies with $[\mathrm{Fe} / \mathrm{H}]$. The results would not change significantly if we assume Eqs. (16) or Eq. (20) instead of Eq. (12). In Fig. 9 we show for instance the comparisons of models for which $\left(\alpha_{\mathrm{O}}\right.$, $\left.\alpha_{\mathrm{Fe}}\right)=(5,2)$ (solid line), $(2,5)$ (dotted line) and $(2,2)$ (dashed line). We show the results for our reference set of parameters $(\lambda=3$ and $\Lambda=1)$ in the left panel. To study the dependence of these curves on the inflow and outflow parameters, we also plot the results for $\lambda=1$ and $\Lambda=3$ (right panel). In order to calculate the yields, we have assumed a Salpeter IMF between 0.1 and $40 M_{\odot}$ and the nucleosynthetic prescriptions of Woosley \& Weaver (1995) for solar metallicity (case B). For the solar abundances we adopt the Anders \& Grevesse (1989) values. We use this set of solar abundances in order to be consistent with the choice of Woosley \& Weaver's (1995) yields.

As expected, as the galaxy ages (and, therefore, $[\mathrm{Fe} / \mathrm{H}]$ increases), $[\mathrm{O} / \mathrm{Fe}]$ bends down if the oxygen ejection efficiency is larger (solid line), whereas it starts increasing if $\alpha_{\mathrm{Fe}}>\alpha_{\mathrm{O}}$ (dotted line). The model with $\lambda=1$ and $\Lambda=3$ shows the same behavior but with reduced variations of the abundance ratios. This is due to the fact that the difference between the metallicities attained for $\alpha=2$ and $\alpha=5$ in the case $(\lambda, \Lambda)=(3,1)$ is larger than in the case $(\lambda, \Lambda)=(1,3)$ (see Figs. 3 and 4$)$. Of course, the model in which the ejection efficiencies are the same (dashed line) does not show any variation of $[\mathrm{O} / \mathrm{Fe}]$ with $[\mathrm{Fe} / \mathrm{H}]$ (see Eq. (29)). The $[\mathrm{O} / \mathrm{Fe}]$ attained by this model $(\sim 0.2)$ is lower than the $[\mathrm{O} / \mathrm{Fe}]$ of halo stars and of the most metal-poor stars of Local Group dwarf galaxies, whose chemical enrichment should have been dominated by SNeII ejecta. Indeed, it is known since quite some time 
that Woosley \& Weaver's yields overpredict iron (Timmes et al. 1995; Chiappini et al. 1997). As we have said, the model with $\lambda=3$ and $\Lambda=1$ can be representative of dwarf spheroidal galaxies. Indeed, in this model the metallicity remains significantly lower than the solar value and, if $\mathrm{O}$ is ejected more easily than $\mathrm{Fe}$, a knee at $[\mathrm{Fe} / \mathrm{H}] \sim-1.5$ appears, in agreement with observations (see e.g. Venn et al. 2004). We point out once more that we are neglecting SNeIa therefore, in the framework of simple models, $\mathrm{O}$ and $\mathrm{Fe}$ are both produced by massive stars, on similar timescales. As a consequence, they should share a similar fate and have comparable ejection efficiencies. Recchi et al. (2004), by means of detailed chemodynamical simulations, found similar values of $\alpha_{\mathrm{O}}$ and $\alpha_{\mathrm{Fe}}$ when complex star formation histories are assumed. Therefore, simple models predict that variations in the $[\mathrm{O} / \mathrm{Fe}]$ vs. $[\mathrm{Fe} / \mathrm{H}]$ plot cannot be attributed to differential winds and SNeIa must necessarily be considered in order to properly interpret this diagram.

Would this conclusion still hold if we relax IRA and take properly into account stellar lifetimes? In order to test that, we have performed a series of numerical simulations of the chemical evolution of a dwarf spheroidal galaxy whose structural parameters resemble those of Local Group such as Carina, Draco, Ursa Minor, Sculptor. In particular we have run a simulation very similar to the "standard" model of Lanfranchi \& Matteucci (2003). The standard model is adjusted to reproduce the average $[\alpha / \mathrm{Fe}]$ and neutron capture abundance ratios of six local dwarf spheroidals. It is assumed that the galaxy forms through the fast and continuous infall of pristine gas until a mass of $\sim 10^{8} M_{\odot}$ is reached. The star formation consists of one long episode ( $8 \mathrm{Gyr}$ ) with low efficiency ( $\left.v=0.05 \mathrm{Gyr}^{-1}\right)$ and is affected by very intense galactic winds (with a rate 2 times higher than the SFR). As the galactic wind develops, it removes a large gas fraction thus decreasing the SFR. This effect, coupled with the low star formation rate and the injection of Fe into the ISM by SNe Ia, gives rise to the knee observed in $[\alpha / \mathrm{Fe}]$ and $[r$-process $/ \mathrm{Fe}]$. The models we show now contain non-selective winds. We plotted the $[\mathrm{O} / \mathrm{Fe}]$ vs. $[\mathrm{Fe} / \mathrm{H}]$ resulting from the standard model in Fig. 10 (solid line). We have then run the same model without SNeIa (dot-short-dashed line) and without SNeIa and without galactic winds (long-dashed line). We have also considered a model without SNeIa in which the ejection efficiency of $\alpha$-elements is enhanced (selective winds) (short-dashed line). Similarly to what we have seen in Fig. 9, if we neglect SNeIa, there is a negligible variation of the $[\mathrm{O} / \mathrm{Fe}]$ ratio as a function of $[\mathrm{Fe} / \mathrm{H}]$ (dot-shortdashed and long-dashed lines) and a knee can be attained only in the presence of $\alpha$-enhanced winds, namely winds in which $\mathrm{O}$ is ejected out of the galaxy more efficiently than Fe $\left(\alpha_{\mathrm{O}}=2 \alpha_{\mathrm{Fe}}\right.$; short-dashed line). The turnoff point of this curve corresponds to the moment in which the galaxy experiences the galactic wind. This plot shows that only a model which takes properly into account SNe Ia and galactic winds is able to produce the knee in $[\alpha / \mathrm{Fe}]$ observed in several dwarf spheroidal galaxies.

\section{Discussion and conclusions}

In this paper we have studied general properties of simple chemical evolution models of galaxies with infall and differential winds, namely with a selective loss of metals from the parent galaxy. We have seen that, assuming gas flows proportional to the SFR, the equations of the evolution of gas mass, total mass and metallicity $\mathrm{Z}$ of gas can be solved analytically, leading to a simple expression of the variation of $\mathrm{Z}$ as a function of the gas mass fraction $\mu$ and of the ejection efficiency of metals $\alpha$ (which is defined as the ratio between the metallicity of the galactic

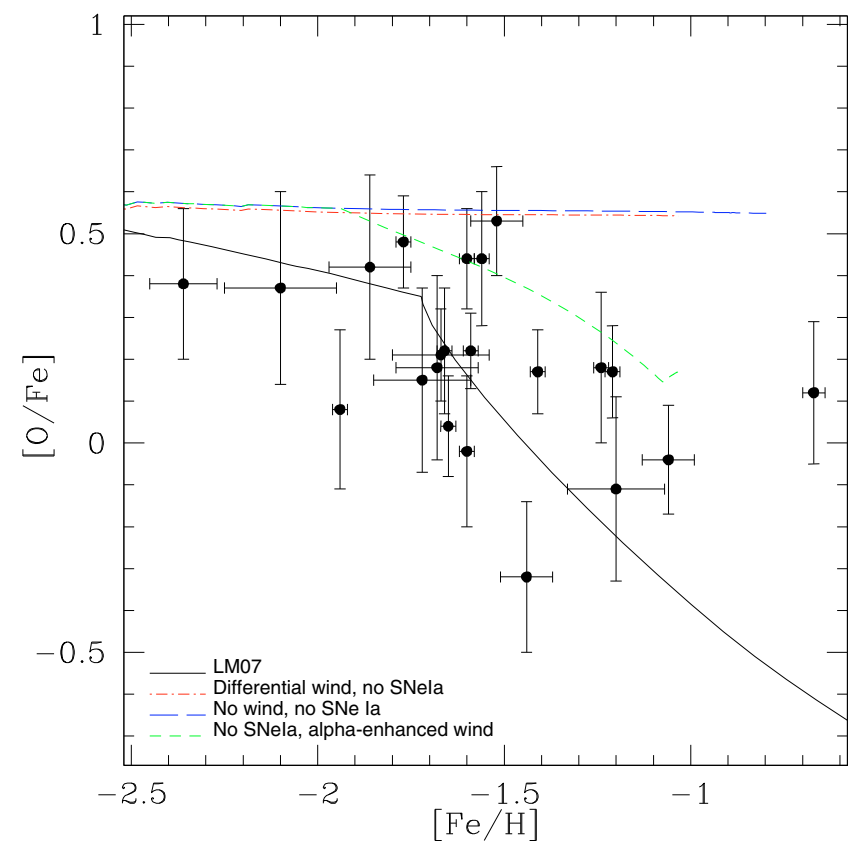

Fig. 10. $[\mathrm{O} / \mathrm{Fe}]$ vs. $[\mathrm{Fe} / \mathrm{H}]$ for numerical models of a standard dwarf spheroidal galaxy. Solid line: wind model like in Lanfranchi \& Matteucci (2007); dot-short-dashed line: the same model without SNeIa; long-dashed line: the same model without SNeIa and without galactic winds; short-dashed line: model without SNeIa in which the ejection efficiency of $\alpha$-elements is enhanced. Data are from Bonifacio et al. (2000, 2004); Shetrone et al. (2001, 2003); Venn et al. (2004); Sadakane et al. (2004); Fulbright et al. (2004); Geisler et al. (2005). For clarity purposes, the dot-short-dashed line has been shifted of $0.01 \mathrm{dex}$ since otherwise it would have been overlapped with the long-dashed line.

wind and the metallicity of the ISM). As expected, the resulting metallicity of the galaxy turns out to be systematically lower than the metallicity attained by models with non-differential winds. In particular, the metallicity of models with differential winds tends asymptotically (for low values of $\mu$, namely during the late evolution of the galaxy) to a constant value which decreases almost linearly with $\alpha$. We have checked that, for exponential infall laws and star formation rates proportional to the gas mass (linear Schmidt law) a constancy between the star formation rate and the infall rate arises quite naturally during the late evolution of the galaxies, provided that the star formation timescale does not differ significantly from the infall timescale. In the first few Gyr the ratio between infall rate and star formation rate can vary quite significantly but, in spite of that, the resulting evolution of the metallicity as a function of time does not change appreciably compared to the case with linear flows.

We have also analyzed models in which the metallicity of the infalling gas varies with time. A realistic case of infalling gas with increasing metallicity can be envisaged in the interaction galactic winds/ICM. Indeed, this interaction must certainly lead to some pollution of the gas external to the galaxy, therefore to a constant increase of the ICM metallicity, in particular when differential winds are considered. In order to take it into account, we have made two extreme assumptions:

- The metals carried out of the galaxy instantaneously and uniformly mix with the surrounding ICM (instantaneous ICM mixing).

- The metallicity of the infalling gas is set to be equal to the metallicity of the outflow, representing a physical situation 
in which the very same gas carried out of the galaxy by outflows (or a fraction of it) falls back to the galaxy due to the pull of its gravitational potential (galactic fountain).

Real physical situations should lie between these two extreme assumptions since the distribution of metals in the ICM is expected to be patchy (Domainko et al. 2006; Sanders \& Fabian 2006; Tornatore et al. 2007). The metallicities predicted by instantaneous ICM mixing models are of course larger than models in which the infalling gas has a pristine chemical composition. The magnitude of this difference depends on the relative amount of ISM and ICM masses. Under normal circumstances, in which the ICM mass is larger than the ISM one, the difference in the final metallicity is only $\sim 0.1-0.2$ dex. A larger ISM metallicity is obtained by galactic fountains models. This result due to the fact that galactic fountains maximize the chemical enrichment of the infalling gas. This metallicity tends however to the one attained by the model with $Z_{A}=0$ if very large metal ejection efficiencies ( $\alpha \gg 1)$ are assumed.

We have then studied the metallicity ratios produced by differential wind models. We have seen that the models with variable infall metallicities (both instantaneous ICM mixing and galactic fountain) simply predict that abundance ratios between chemical elements are given by the ratio of their respective stellar yields (if these do not change with metallicity). Assuming a fixed infall metallicity $Z_{A} \neq 0$ this ratio also depends on the value of $Z_{A}$ and on the infall parameter $\Lambda$, but nevertheless it does not change during the evolution of the galaxy. However, we stress here that simple models can be properly applied only to $\mathrm{SNeII}$ products, whose release is very prompt. Iron is for instance mostly produced by SNeIa, whose explosion timescales range from a few tens of Myr to several Gyr, depending on the star formation history of the considered galaxy (Matteucci \& Recchi 2001), therefore only the fraction of iron produced by SNeII ( $\sim 1 / 3$ of the total) can be treated by the simple models. A constant $\mathrm{O} / \mathrm{Fe}$ ratio, as predicted by simple models is therefore expected because, in this case, both chemical elements are produced by massive stars, on similar timescales and they should therefore have a similar evolution. However, $\mathrm{O} / \mathrm{Fe}$ ratios are not constant in galaxies and larger ratios are observed in older, more metal-poor stars. The most natural explanation of this trend is that the delayed production of iron by SNeIa reduces the initially high $\mathrm{O} / \mathrm{Fe}$ ratio of the oldest stars (time-delay model, Matteucci \& Greggio 1986).

Simple models can produce variable abundance ratios either if we assume different ejection efficiencies for different chemical species (selective winds) or an IMF variable in time. Both assumptions should be quite "ad hoc". We have tested the case of selective winds for the $\mathrm{O} / \mathrm{Fe}$ ratio showing that, if the $\mathrm{O}$ ejection efficiency is significantly larger than the $\mathrm{Fe}$ one, $\mathrm{O} / \mathrm{Fe}$ decreases with time (and with $\mathrm{Fe} / \mathrm{H}$ ). We have confirmed this result also by means of detailed numerical simulations in which IRA is relaxed showing that, neglecting SNeIa, the only way to get a knee in the $[\mathrm{O} / \mathrm{Fe}]$ vs. $[\mathrm{Fe} / \mathrm{H}]$ relation is by assuming $\alpha$-enhanced galactic winds. In a model suitable for dwarf spheroidal galaxies, in which the outflow rate exceeds the infall rate, the $\mathrm{O} / \mathrm{Fe}$ is almost constant up to $[\mathrm{Fe} / \mathrm{H}] \sim-1.5$, then it bends down. This behavior is consistent with the observation of many dwarf spheroidal galaxies of the Local Group (Venn et al. 2004, and references therein). If instead iron is ejected more efficiently than oxygen, the opposite happens, with $\mathrm{O} / \mathrm{Fe}$ ratios increasing with time. On the other hand, an iron ejection efficiency larger than the oxygen seems plausible, as it has been shown by chemodynamical simulations of dwarf galaxies experiencing violent bursts of star formation (Recchi et al. 2001, 2002). However, also these models predict $\mathrm{O} / \mathrm{Fe}$ ratios decreasing with time (and with $[\mathrm{Fe} / \mathrm{H}]$ ) because SNeIa were not neglected and their contribution is more important than selective winds in determining the evolution of the $\mathrm{O} / \mathrm{Fe}$ ratio. Moreover, Recchi et al.'s model found larger iron ejection efficiencies precisely because of the delayed explosion of SNeIa. These SNe explode in a medium already heated and diluted by the previous activity of SNeII, therefore SNeIa products can be very easily channeled along the galactic funnel. If we neglect $\mathrm{SNeIa}, \mathrm{O}$ and $\mathrm{Fe}$ are produced by the same stars and they should therefore share the same fate.

Our main conclusions can be summarized as follows:

- The differential winds have the effect of reducing the global metallicity of a galaxy. The magnitude of this reduction increases with the ejection efficiency of metals.

- This remains true also if we allow a variable metallicity of the infalling gas. Larger galactic metallicities are obtained if we assume that the infall has the metallicity of the outflowing gas (galactic fountain model).

- Simple models with constant (i.e. non metallicitydependent) yields predict constant abundance ratios, even in the presence of differential winds or variable infall metallicities. The same occurs for detailed numerical models.

- One way to get variable abundance ratios in simple models is by assuming selective winds, namely differential winds with different ejection efficiencies for each chemical element. However, simple models apply only to chemical elements promptly released on short timescales, therefore large element-to-element variations of the ejection efficiencies are not very likely. Abundance ratios predicted by simple models of chemical evolution are then likely to remain constant during the evolution of the galaxy.

Acknowledgements. We thank the anonymous referee whose comments and criticisms have improved the quality of the paper. We thank F. Calura, G. Cescutti and A. Palestini for important support and stimulating discussions. G.A.L. acknowledges financial support from the Brazilian agency FAPESP (Proj. 06/57824-1).

\section{Appendix A}

Here we give the explicit value of some constants we have used in Sect. 3.4.

$E_{1}=(\lambda+1)(1-R) S t_{\mathrm{i}}-1$.

$E_{2}=\lambda(\alpha-1)(1-R) S t_{\mathrm{i}}$

$K_{1}=\gamma E_{1}-1$

$K_{2}=\frac{K_{1}}{\lambda(\alpha-1)}$

$K_{3}=\frac{(1-R) S t_{\mathrm{i}}}{(1-R)(1+\alpha \lambda) S t_{\mathrm{i}}-1}$

\section{References}

Anders, E., \& Grevesse, N. 1989, Geochim. Cosmochim. Acta, 53, 197

Balestra, I., Tozzi, P., Ettori, S., et al. 2007, A\&A, 462, 429

Bonifacio, P., Hill, V., Molaro, P., et al. 2000, A\&A, 359, 663

Bonifacio, P., Sbordone, L., Marconi, G., Pasquini, L., \& Hill, V. 2004, A\&A, 414,503

Bregman, J. N. 1980, ApJ, 365, 544

Calura, F., Matteucci, F., \& Tozzi, P. 2007, MNRAS, 378, L11 
Cescutti, G., Matteucci, F., François, P., \& Chiappini, C. 2007, A\&A, 462, 943 Chiappini, C., Matteucci, F., \& Gratton, R. 1997, ApJ, 477, 765 Clayton, D. D. 1988, MNRAS, 234, 1

Colavitti, E., Matteucci, F., \& Murante, G. 2008, A\&A, 483, 401 Dekel, A., \& Silk, J. 1986, ApJ, 303, 39

D'Ercole, A., \& Brighenti, F. 1999, MNRAS, 309, 941

Domainko, W., Mair, M., Kapferer, W., et al. 2006, A\&A, 452, 795

Edmunds, M. G. 1990, MNRAS, 246, 678

Fujita, A., MacLow, M.-M., Ferrara, A., \& Meiksin, A. 2004, ApJ, 613, 159

Fulbright, J. P., Rich, R. M., \& Castro, S. 2004, ApJ, 612, 447

Garnett, D. R. 2002, ApJ, 581, 1019

Geisler, D., Smith, V. V., Wallerstein, G., Gonzalez, G., \& Charbonnel, C. 2005, AJ, 129, 1428

Greggio, L., \& Renzini, A. 1983, A\&A, 118, 217

Hartwick, F. D. A. 1976, ApJ, 209, 418

Heckman, T. 2002, in Extragalactic Gas at Low Redshift (San Francisco: ASP), ed. J. Mulchaey, \& J. Stocke, ASP Conf. Ser., 254, 292

Köppen, J. 1994, A\&A, 281, 26

Köppen, J., \& Edmunds, M. G. 1999, MNRAS, 306, 317

Lanfranchi, G. A., \& Matteucci, F. 2003, MNRAS, 345, 71

Lanfranchi, G. A., \& Matteucci, F. 2004, MNRAS, 351, 1338

Lanfranchi, G. A., \& Matteucci, F. 2007, A\&A, 468, 927

Larson, R. B. 1976, MNRAS, 176, 31

Lynden-Bell, D. 1975, Vistas Astron., 19, 299

MacLow, M.-M., \& Ferrara, A. 1999, ApJ, 513, 142

Mannucci, F., Della Valle, M., Panagia, N., et al. 2005, A\&A, 433, 807

Mannucci, F., Della Valle, M., \& Panagia, N. 2006, MNRAS, 370, 773

Marconi, G., Matteucci, F., \& Tosi, M. 1994, MNRAS, 270, 35

Martin, C. L., Kobulnicky, H. A., \& Heckman, T. M. 2002, ApJ, 574, 663

Martinelli, A., \& Matteucci, F. 2000, A\&A, 353, 269

Mathews, W. G., \& Baker, J. C. 1971, ApJ, 170, 241

Matteucci, F. 2001, The Chemical Evolution of the Galaxy, ASSL (Kluwer Academic Publisher)
Matteucci, F., \& Chiappini, C. 2005, PASA, 22, 49

Matteucci, F., \& Greggio, L. 1986, A\&A, 154, 279

Matteucci, F., \& Recchi, S. 2001, ApJ, 558, 351

Matteucci, F., \& Vettolani, G. 1988, A\&A, 202, 21

Moretti, A., Portinari, L., \& Chiosi, C. 2003, A\&A, 408, 431

Ott, J., Walter, F., \& Brinks, E. 2005, MNRAS, 358, 1453

Pagel, B. E. J., \& Patchett, B. E. 1975, MNRAS, 172, 13

Pagel, B. E. J., \& Tautvaišienè, G. 1998, MNRAS, 299, 535

Pilyugin, L. S. 1993, A\&A, 277, 42

Prantzos, N., \& Aubert, O. 1995, A\&A, 302, 69

Rana, N. C. 1991, ARA\&A, 29, 129

Recchi, S., Matteucci, F., \& D’Ercole, A. 2001, MNRAS, 322, 800

Recchi, S., Matteucci, F., D’Ercole, A., \& Tosi, M. 2002, A\&A, 384, 799

Recchi, S., Matteucci, F., D’Ercole, A., \& Tosi, M. 2004, A\&A, 426, 37

Sadakane, K., Arimoto, N., Ikuta, C., et al. 2004, PASJ, 56, 1041

Salvadori, S., Ferrara, A., \& Schneider, R. 2008, MNRAS, 386, 348

Sanders, J. S., \& Fabian, A. C. 2006, MNRAS, 371, 1483

Schmidt, M. 1963, ApJ, 137, 758

Searle, L., \& Sargent, W. L. W. 1972, ApJ, 173, 25

Shapiro, P. R., \& Field, G. B. 1976, ApJ, 205, 762

Shetrone, M. D., Côté, P., \& Sargent, W. L. W. 2001, ApJ, 548, 592

Shetrone, M. D., Venn, K. A., Tolstoy, E., et al. 2003, AJ, 125, 684

Silk, J. 2003, MNRAS, 343, 249

Skillman, E. D. 1997, Rev. Mex. AC, 6, 36

Spitoni, E., Recchi, S., \& Matteucci, F. 2008, A\&A, 484, 743

Timmes, F. X., Woosley, S. E., \& Weaver, T. A. 1995, ApJS, 98, 617

Tinsley, B. M. 1974, ApJ, 192, 629

Tinsley, B. M. 1980, Fund. Cosmic Phys., 5, 287

Tornatore, L., Borgani, S., Dolag, K., \& Matteucci, F. 2007, MNRAS, 382, 1050 Tremonti, C. A., Heckman, T. M., Kauffmann, G., et al. 2004, ApJ, 613, 898

Twarog, B. 1980, ApJ, 242, 242

Venn, K. A., Irwin, M., Shetrone, M. D., et al. 2004, AJ, 128, 1177

Woosley, S. E., \& Weaver, T. A. 1995, ApJS, 101, 181 